László Zsilinszky, Department of Mathematics, University of South Carolina, Columbia, SC 29208, e-mail: zsilinsz@math.sc.edu

\title{
SUPERPOROSITY IN A CLASS OF NON-NORMABLE SPACES
}

\section{INTRODUCTION}

The concept of porous set was introduced by Dolženko in [D]. Since then it has been thoroughly investigated and diversely generalized (see [Za1] or [Re] for a survey). It is possible to define several notions concerning porosity also in metric spaces (see [Za1],[Re]). It is known that in Banach spaces the ideal of meager sets is strictly wider than that of the $\sigma$-porous sets ([Za1]). It is true also in closed non-locally compact convex subsets of a separable Banach space ([AB]). Recently it has been established in dense in itself completely metrizable spaces as well (cf. $[\mathrm{Za} 3])$.

The primary goal of the research presented in this paper is in the line of the above results, i.e. to compare $\sigma$-porous and meager sets, respectively in some nonnormable spaces. Such an attempt was made in [TZs] where the space $s$ of all real sequences endowed with the Fréchet metric

$$
\rho_{F}\left(\left\{a_{n}\right\}_{n},\left\{b_{n}\right\}_{n}\right)=\sum_{n} 2^{-n} \frac{\left|a_{n}-b_{n}\right|}{1+\left|a_{n}-b_{n}\right|} \text { where }\left\{a_{n}\right\}_{n},\left\{b_{n}\right\}_{n} \in s
$$

was scrutinized in this respect. This space is non-normable ([KG], Exercise 276) and it was shown in [TZs] e.g. that the set $\left\{\left\{a_{n}\right\}_{n} \in s ; \sum_{n} \Phi\left(a_{n}\right)\right.$ converges $\}$ is $\sigma$ superporous in $s$ for a residual family of functions $\Phi$ in the space of all real functions furnished with the uniform topology.

It is the purpose of this paper to carry on these investigations generalizing results of [TZs] for the space $\mathcal{M}$ of all measurable functions on an infinite $\sigma$-finite measure space $(X, S, \mu)$ endowed with the (metrizable) topology of convergence in measure on sets of finite measure (see $[G]$ ). We will show that results quite analogous to those of exposed in [TZs] for $s$ hold in this generality as well. For instance, the set $A(\Phi)=\left\{f \in \mathcal{M} ; \int_{X}^{*}|\Phi \circ f| d \mu^{*}<+\infty\right\}$ is $\sigma$-superporous in $\mathcal{M}$ for a broad class of functions $\Phi: \mathbb{R} \rightarrow \mathbb{R}$, where $\mu^{*}$ is the outer measure induced by $\mu$ and $\int_{X}^{*} h d \mu^{*}$ stands for the $\mu^{*}$-upper integral of the function $h: X \rightarrow \mathbb{R}$ (see $[\mathrm{F}]$, Section 2.4).

Further we show that $A\left(\chi_{\mathbb{R} \backslash M}\right)$ is $\sigma$-superporous in $\mathcal{M}$ for every $\sigma$-very porous set $M \subset \mathbb{R} \quad\left(\chi_{\mathbb{R} \backslash M}\right.$ is the characteristic function of $\left.\mathbb{R} \backslash M\right)$ and that $A\left(\chi_{\mathbb{R} \backslash M}\right)$ is meager in $\mathcal{M}$ if $M$ is meager at some point of $\mathbb{R}$. In particular, $A\left(\chi_{\mathbb{R} \backslash M}\right)$ is meager in $\left(s, \rho_{F}\right)$ if and only if $M$ is meager at some point of $\mathbb{R}$.

This could provide a method for relating meager non- $\sigma$-porous subsets of $\mathbb{R}$ to meager non- $\sigma$-porous subsets of $\mathcal{M}$ (resp. $s$ ) if the porosity of $A\left(\chi_{\mathbb{R} \backslash M}\right)$ in $\mathcal{M}$ (resp.

$s$ ) could be characterized in terms of $M \subset \mathbb{R}$.

It is worth noticing here that a more familiar metrization of $\mathcal{M}$ by the metric

$$
m(f, g)=\inf \{\varepsilon>0 ; \mu(\{x \in X ;|f(x)-g(x)| \geq \varepsilon\})<\varepsilon\}(f, g \in \mathcal{M})
$$

Typeset by $\mathcal{A M}_{\mathcal{M}}-\mathrm{T}_{\mathrm{E}} \mathrm{X}$ 
which coincides with the topology of convergence in measure on $X$ (cf. [F], Section 2.3.8), yields a setting where our considerations are not feasible even for continuous $\Phi ' s$. This question was studied in [Zs1].

\section{Preliminaries}

In the sequel $(X, S, \mu)$ will be an infinite $\sigma$-finite measure space and $\mu^{*}$ the outer measure induced by $\mu$. Without loss of generality we may suppose that $X=\bigcup_{n=1}^{\infty} X_{n}$, where $\left\{X_{n}\right\}_{n=1}^{\infty}$ is a sequence of pairwise disjoint, $S$-measurable sets such that $2<\mu\left(X_{n}\right)<+\infty$ for each $n \in \mathbb{N}$.

Denote by $\mathcal{M}$ (resp. $\mathcal{M}_{n}$ ) the set of all $S$-measurable functions that are finite almost everywhere (abbr. a.e.) on $X$ (on $X_{n}$ ). We will identify members of $\mathcal{M}$ provided they equal a.e. on $X$. If the sequence $f_{k} \in \mathcal{M}(k \in \mathbb{N})$ converges in measure to $f \in \mathcal{M}$, write $f_{k} \stackrel{\mu}{\longrightarrow} f$.

Denote by $\mathcal{F}_{m}$ the space of all functions $\Phi: \mathbb{R} \rightarrow \mathbb{R}$ such that $\Phi \circ f \in \mathcal{M}$ for all $f \in \mathcal{M}$. It is known that $\mathcal{F}_{m}$ contains the class of Borel-measurable functions. Observe that $\mathcal{F}_{m}$ is a closed subspace of the complete metric space $(\mathcal{F}, d)$, where $\mathcal{F}=\mathbb{R}^{\mathbb{R}}$ and

$$
d(\Phi, \Psi)=\min \left\{1, \sup _{t \in \mathbb{R}}|\Phi(t)-\Psi(t)|\right\} \quad(\Phi, \Psi \in \mathcal{F}) .
$$

Indeed, if a sequence $\Phi_{n} \in \mathcal{F}_{m}(n \in \mathbb{N}) \quad d$-converges to $\Phi \in \mathcal{F}$ then $\Phi_{n} \circ f \in \mathcal{M}$ converges pointwise to $\Phi \circ f$ (for all $f \in \mathcal{M}$ ), thus $\Phi \circ f \in \mathcal{M}$ and consequently $\Phi \in \mathcal{F}_{m}$. It follows that $\left(\mathcal{F}_{m}, d\right)$ is a complete metric space.

For $\Phi \in \mathcal{F}$ and $p \in \mathbb{N}$ define

$$
\begin{gathered}
A(\Phi)=\left\{f \in \mathcal{M} ; \int_{X}^{*}|\Phi \circ f| d \mu^{*}<+\infty\right\} \text { and } \\
A_{p}(\Phi)=\left\{f \in \mathcal{M} ; \int_{X}^{*}|\Phi \circ f| d \mu^{*} \leq p\right\}
\end{gathered}
$$

where $\int_{X}^{*} f d \mu^{*}$ is the upper integral of $f$ with respect to $\mu^{*}$ (see [F], Section 2.4).

For $f, g \in \mathcal{M}$ and $n \in \mathbb{N}$ define

$$
\begin{gathered}
\rho_{n}(f, g)=\int_{X_{n}} \frac{|f-g|}{1+|f-g|} d \mu \\
\rho(f, g)=\sum_{n=1}^{\infty} \frac{1}{2^{n} \mu\left(X_{n}\right)} \rho_{n}(f, g) .
\end{gathered}
$$

For $i, j \in \mathbb{N}$ and $M \subset \mathbb{R}$ denote

$$
\begin{gathered}
\tilde{A}_{i, j}(M)=\left\{f \in \mathcal{M} ; \mu^{*}\left(f^{-1}(M) \cap X_{i}\right) \geq \frac{\mu\left(X_{i}\right)}{j}\right\} \\
A_{i, j}(M)=\left\{\left.f\right|_{X_{i}} ; f \in \tilde{A}_{i, j}(M)\right\} \text { and } A_{i, 0}(M)=\left\{f \in \mathcal{M}_{i} ; \mu^{*}\left(f^{-1}(M)\right)=\mu\left(X_{i}\right)\right\} .
\end{gathered}
$$

It is not hard to see that $\rho$ (resp. $\rho_{n}$ ) is a metric on $\mathcal{M}$ (resp. $\mathcal{M}_{n}$ ). It can be shown similarly as for $\left(s, \rho_{F}\right)$ that $(\mathcal{M}, \rho)$ is non-normable (see [Zs2]).

Convergence in measure implies $\rho$-convergence and the converse holds if and only if the underlying measure space is finite. More precisely we have: 
Lemma 1. Let $f_{k}, f \in \mathcal{M}(k \in \mathbb{N})$. The following are equivalent:

(i) $f_{k} \stackrel{\rho}{\rightarrow} f$;

(ii) $f_{k} \stackrel{\mu}{\longrightarrow} f$ on every $S$-measurable set of finite measure;

(iii) $\left.\left.f_{k}\right|_{X_{n}} \stackrel{\rho_{n}}{\longrightarrow} f\right|_{X_{n}}$ for all $n \in \mathbb{N}$.

Proof. For (i) $\Leftrightarrow$ (ii) see [G], Theorem 3. The equivalence (i) $\Leftrightarrow$ (iii) follows easily from $[\mathrm{K}]$ (Theorem 14, p.122).

Remark 1. Observe that $\left(\mathcal{M}_{n}, \rho_{n}\right)$ is a complete metric space for each $n \in \mathbb{N}$ and the $\rho_{n}$-convergence of sequences from $\mathcal{M}_{n}$ coincides with their convergence in measure on $X_{n}$ ([Ha], Problem 42(4)). Further the equivalence (i) $\Leftrightarrow$ (iii) in the previous lemma actually yields that $(\mathcal{M}, \rho)$ and the Tychonoff product $\Pi_{n}\left(\mathcal{M}_{n}, \rho_{n}\right)$ are homeomorphic.

Lemma 2. (cf.[G]) $(\mathcal{M}, \rho)$ is a complete metric space.

Denote by $B_{d}(y, r)$ the open ball about $y \in Y$ with radius $r>0$ in the metric space $(Y, d)$. By $B(x, r)$ we will denote the interval $(x-r, x+r)$, where $x \in \mathbb{R}$. For $E \subset Y, y \in Y$ and $r>0$ define

$$
\gamma(y, r, E)=\sup \left\{r^{\prime}>0 ; \exists y^{\prime} \in Y \quad B_{d}\left(y^{\prime}, r^{\prime}\right) \subset B_{d}(y, r) \backslash E\right\} .
$$

We say that $E$ is porous (very porous) at $y$ if

$$
\limsup _{r \rightarrow 0^{+}} \frac{\gamma(y, r, E)}{r}>0\left(\liminf _{r \rightarrow 0^{+}} \frac{\gamma(y, r, E)}{r}>0\right) .
$$

Further $E$ is said to be superporous at $y \in Y$ (see [Za1],[Za2]), if $E \cup F$ is porous at $y$ whenever $F \subset Y$ is porous at $y$.

A set $E \subset Y$ is said to be globally very porous if there exist constants $0<a_{E}<1$ and $r_{0}>0$ such that $\gamma(y, r, E)>a_{E} r$ for every $y \in E$ and $0<r<r_{0}([\mathrm{Za} 1])$.

We say that $E$ is superporous (very porous) if it is superporous (very porous) at each of its points, further $E$ is $\sigma$-superporous ( $\sigma$-very porous) if it is a countable union of superporous (very porous) sets. Superporosity was defined in [Za2] in connection with the $\mathcal{I}$-density topology of Wilczynski and others (cf.[W]).

Note that superporosity implies very porosity as observed in [Za2] (see [Re], Corollary 8.15 as well) and $\sigma$-superporosity is equivalent to $\sigma$-very porosity which is further equivalent to $\sigma$-globally very porosity ([Re], Corollary 8.17).

We will denote by $\operatorname{card} Y$ and $\mathcal{P}(Y)$ the cardinality and the power set, respectively of the set $Y$, further $c$ will stand for the power of the continuum. Denote by $|I|$ the length of the interval $I \subset \mathbb{R}$.

\section{Main Results}

Lemma 3. Let $\left\{I_{q} ; q \in \mathbb{N}\right\}$ be an enumeration of open intervals with rational endpoints. Let $\Phi_{p q}=p \chi_{I_{q}}$ for $p, q \in \mathbb{N}$. Then $A_{p}\left(\Phi_{p q}\right)$ is superporous in $(\mathcal{M}, \rho)$ for every $p, q \in \mathbb{N}$.

Proof. Choose $p, q \in \mathbb{N}$ and denote by $t_{q}$ the midpoint of $I_{q}$. Let $f \in A_{p}\left(\Phi_{p q}\right)$. Suppose that $F \subset \mathcal{M}$ is an arbitrary set porous at $f$. Then there exist sequences 
$r_{n}, r_{n}^{\prime}>0(n \in \mathbb{N})$ and $\alpha>0$ such that $\alpha r_{n}<r_{n}^{\prime}<r_{n}<2^{-n}$, further we get an $f_{n} \in \mathcal{M}$ such that

$$
B_{\rho}\left(f_{n}, r_{n}^{\prime}\right) \subset B_{\rho}\left(f, r_{n}\right) \backslash F .
$$

Define $p_{n}=\min \left\{k \in \mathbb{N} ; 2^{-k}<r_{n}^{\prime}\right\}+1$ and $\varepsilon_{n}=2^{-p_{n}+1}$ for all $n \in \mathbb{N}$. Then we have

$$
r_{n}^{\prime}>\varepsilon_{n} \geq \frac{r_{n}^{\prime}}{2} .
$$

Denote $E_{n 1}=X_{p_{n}} \cap f_{0}^{-1}\left(\left(t_{q}-\frac{1}{8}\left|I_{q}\right|, t_{q}+\frac{1}{8}\left|I_{q}\right|\right)\right)$ and $E_{n 2}=X_{p_{n}} \backslash E_{n 1}$ and define $g_{n}=f_{n} \chi_{X \backslash X_{p_{n}}}+t_{q} \chi_{E_{n 2}}+\left(t_{q}+\frac{1}{4}\left|I_{q}\right|\right) \chi_{E_{n 1}} \in \mathcal{M}$. It is clear that

$$
\left|f_{n}(x)-g_{n}(x)\right| \geq \frac{1}{8}\left|I_{q}\right| \text { for all } x \in X_{p_{n}} .
$$

Since $\rho\left(f_{n}, g_{n}\right)=\frac{1}{2^{p_{n}} \mu\left(X_{p_{n}}\right)} \int_{X_{p_{n}}} \frac{\left|f_{n}-g_{n}\right|}{1+\left|f_{n}-g_{n}\right|} d \mu$ then by the definition of $\varepsilon_{n}, X_{p_{n}}$ and $(3)$, respectively we get

$$
\begin{array}{r}
\rho\left(f_{n}, g_{n}\right)<\frac{\varepsilon_{n}}{2}, \\
\rho\left(f_{n}, g_{n}\right)>\frac{\left|I_{q}\right|}{8+\left|I_{q}\right|} \cdot \frac{\varepsilon_{n}}{2} .
\end{array}
$$

Put $\delta_{n}=\frac{\left|I_{q}\right|}{16+2\left|I_{q}\right|} \rho\left(f_{n}, g_{n}\right)$ and pick an arbitrary $h_{n} \in B_{\rho}\left(g_{n}, \delta_{n}\right)$. Define

$$
D_{n}=\left\{x \in X_{p_{n}} ;\left|h_{n}(x)-g_{n}(x)\right|<\frac{4 \delta_{n}}{\varepsilon_{n}-4 \delta_{n}}\right\} \text { and } D_{n 0}=X_{p_{n}} \backslash D_{n} .
$$

Observe that $D_{n}$ is well-defined, since by (4) $\delta_{n}=\frac{\left|I_{q}\right|}{8+\left|I_{q}\right|} \cdot \frac{\rho\left(f_{n}, g_{n}\right)}{2}<\frac{\varepsilon_{n}}{4}$.

Then we have

$$
\begin{gathered}
\delta_{n}>\rho\left(h_{n}, g_{n}\right) \geq \frac{1}{2^{p_{n}} \mu\left(X_{p_{n}}\right)} \int_{D_{n 0}} \frac{\left|h_{n}-g_{n}\right|}{1+\left|h_{n}-g_{n}\right|} d \mu \geq \\
\geq \frac{\varepsilon_{n}}{2 \mu\left(X_{p_{n}}\right)} \int_{D_{n 0}} \frac{4 \delta_{n}}{\varepsilon_{n}} d \mu=\frac{2 \delta_{n} \mu\left(D_{n 0}\right)}{\mu\left(X_{p_{n}}\right)},
\end{gathered}
$$

thus $\mu\left(D_{n 0}\right)<\frac{1}{2} \mu\left(X_{p_{n}}\right)$ hence $\mu\left(D_{n}\right) \geq \frac{1}{2} \mu\left(X_{p_{n}}\right)>1$.

In view of (4) we get $\left|h_{n}(x)-g_{n}(x)\right|<\frac{4 \delta_{n}}{\varepsilon_{n}-4 \delta_{n}}<\frac{1}{8}\left|I_{q}\right|$ for every $x \in D_{n}$, so $h_{n}\left(D_{n}\right) \subset\left(t_{q}-\frac{3}{8}\left|I_{q}\right|, t_{q}+\frac{3}{8}\left|I_{q}\right|\right)$ (see the definition of $\left.g_{n}\right)$. Then $\int_{X}^{*}\left|\Phi_{p q} \circ h_{n}\right| d \mu^{*} \geq$ $\int_{D_{n}}^{*}\left|\Phi_{p q} \circ h_{n}\right| d \mu^{*} \geq p \mu\left(D_{n}\right)>p$, so

$$
h_{n} \in \mathcal{M} \backslash A_{p}\left(\Phi_{p q}\right) .
$$

Using (4) we get $\varepsilon_{n}-\rho\left(f_{n}, g_{n}\right)>\frac{\varepsilon_{n}}{2}>\frac{\varepsilon_{n}}{2} \cdot \frac{\left|I_{q}\right|}{8+\left|I_{q}\right|}>\delta_{n}$, therefore $B_{\rho}\left(g_{n}, \delta_{n}\right) \subset$ $B_{\rho}\left(f_{n}, \varepsilon_{n}\right) \subset B_{\rho}\left(f_{n}, r_{n}^{\prime}\right)$. Then in virtue of (5) and (1) there holds

$$
B_{\rho}\left(g_{n}, \delta_{n}\right) \subset B_{\rho}\left(f_{n}, r_{n}^{\prime}\right) \backslash A_{p}\left(\Phi_{p q}\right) \subset B_{\rho}\left(f, r_{n}\right) \backslash\left(F \cup A_{p}\left(\Phi_{p q}\right)\right) .
$$

From (4') and (2) we get

$$
\gamma\left(f, r_{n}, F \cup A_{p}\left(\Phi_{p q}\right)\right) \geq \delta_{n} \geq\left(\frac{\left|I_{q}\right|}{8+\left|I_{q}\right|}\right)^{2} \frac{\varepsilon_{n}}{2} \geq\left(\frac{\left|I_{q}\right|}{8+\left|I_{q}\right|}\right)^{2} \frac{r_{n}^{\prime}}{4}>\left(\frac{\left|I_{q}\right|}{8+\left|I_{q}\right|}\right)^{2} \frac{\alpha}{4} r_{n},
$$

thus $\limsup _{r \rightarrow 0^{+}} \frac{\gamma\left(f, r, F \cup A_{p}\left(\Phi_{p q}\right)\right)}{r} \geq\left(\frac{\left|I_{q}\right|}{8+\left|I_{q}\right|}\right)^{2} \frac{\alpha}{4}>0$, which proves the porosity of $F \cup$ $A_{p}\left(\Phi_{p q}\right)$ at $f$. 
Theorem 1. Let $\Phi \in \mathcal{F}$ be a function for which there exists $t_{0} \in \mathbb{R} \cup\{ \pm \infty\}$ such that

$$
\liminf _{t \rightarrow t_{0}}|\Phi(t)|>0
$$

Then $A(\Phi)$ is $\sigma$-superporous in $(\mathcal{M}, \rho)$.

Proof. In view of (6) there exists $\beta>0$ and a bounded open interval $I$ such that

$$
|\Phi(t)| \geq \beta \text { for all } t \in I
$$

Let $\left\{J_{k} ; k \in \mathbb{N}\right\}$ be a partition of $I$ consisting of open intervals. Choose an $f \in A(\Phi)$. Then by (7) we have

$$
\beta \sum_{k \in \mathbb{N}} \mu\left(f^{-1}\left(J_{k}\right)\right)=\beta \mu\left(f^{-1}(I)\right) \leq \int_{X}^{*}|\Phi \circ f| d \mu^{*}<p
$$

for some $p \in \mathbb{N}$. Thus $\mu\left(f^{-1}\left(J_{k}\right)\right) \leq 1$ for some $k \in \mathbb{N}$ and hence $\mu\left(f^{-1}\left(I_{q}\right)\right) \leq 1$ for some open interval $I_{q} \subset J_{k}$ with rational endpoints. Consequently,

$$
\int_{X}^{*}\left|\Phi_{p q} \circ f\right| d \mu^{*}=p \mu\left(f^{-1}\left(I_{q}\right)\right) \leq p
$$

so $f \in A_{p}\left(\Phi_{p q}\right)$, whence $A(\Phi) \subset \bigcup_{p, q \in \mathbb{N}} A_{p}\left(\Phi_{p q}\right)$, which concludes the proof by Lemma 3.

As the following results show, there are also functions $\Phi$, not necessarily satisfying (6), for which $A(\Phi)$ is still $\sigma$-superporous (cf. Theorem 2):

Lemma 4. Let $M \subset \mathbb{R}$ be a globally very porous set. Then $\tilde{A}_{i, j}(M)$ is superporous in $(\mathcal{M}, \rho)$ for each $i, j \in \mathbb{N}$.

Proof. According to the assumption on $M$ there exist $0<a_{M}<1$ and $r_{0}>0$ such that

$$
\gamma(x, r, M)>a_{M} r \text { for all } x \in M \cup(\mathbb{R} \backslash \bar{M}) \text { and all } 0<r<r_{0} \text {. }
$$

Choose $f \in \tilde{A}_{i j}(M)$ and a set $F \subset \mathcal{M}$ which is porous at $f$. Then there exist $\alpha>0$, sequences $r_{n}, r_{n}^{\prime}>0$ and $f_{n} \in \mathcal{M}$ such that $r_{n} \searrow 0, \alpha r_{n}<r_{n}^{\prime}<r_{n}<$ $2^{-i+1} \cdot \frac{3 r_{0}}{1+r_{0}}$ and

$$
B\left(f_{n}, r_{n}^{\prime}\right) \subset B\left(f, r_{n}\right) \backslash F
$$

It is not hard to find $b_{n k} \in \mathbb{R}\left(1 \leq k \leq m_{n}\right.$, where $\left.m_{n} \in \mathbb{N}\right)$ and a partition $\left\{D_{n k} ; 1 \leq k \leq m_{n}\right\}$ of $X_{i}$ such that for $g_{n 0}=f_{n} \chi_{X \backslash X_{i}}+\sum_{k=1}^{m_{n}} b_{n k} \chi_{D_{n k}} \in \mathcal{M}$ there holds

$$
\rho\left(f_{n}, g_{n 0}\right)<\frac{r_{n}^{\prime}}{4}
$$


We can actually choose $b_{n k} \in M \cup(\mathbb{R} \backslash \bar{M})$ for every $1 \leq k \leq m_{n}$.

Put $\eta_{n}=\frac{2^{i} r_{n}^{\prime}}{6-2^{i} r_{n}^{\prime}}$. Then $\eta_{n}<r_{0}$, so it follows from (8) that for each $1 \leq k \leq m_{n}$ there exists $b_{n k}^{\prime} \in \mathbb{R}$ and $r_{n k}>0$ such that

$$
a_{M} \eta_{n} \leq r_{n k}<\eta_{n} \text { and } B\left(b_{n k}^{\prime}, r_{n k}\right) \subset B\left(b_{n k}, \eta_{n}\right) \backslash M
$$

Define $g_{n}=g_{n 0} \chi_{X \backslash X_{i}}+\sum_{k=1}^{m_{n}} b_{n k}^{\prime} \chi_{D_{n k}} \in \mathcal{M}$. Then by (11) we have

$$
\begin{gathered}
\rho\left(g_{n 0}, g_{n}\right) \leq \frac{1}{2^{i} \mu\left(X_{i}\right)} \sum_{k=1}^{m_{n}}\left(\int_{D_{n k}}\left|b_{n k}-b_{n k}^{\prime}\right| d \mu\right)=\frac{1}{2^{i} \mu\left(X_{i}\right)} \sum_{k=1}^{m_{n}}\left|b_{n k}-b_{n k}^{\prime}\right| \mu\left(D_{n k}\right) \leq \\
\leq \frac{1}{2^{i} \mu\left(X_{i}\right)} \eta_{n} \sum_{k=1}^{m_{n}} \mu\left(D_{n k}\right)=\frac{r_{n}^{\prime}}{6-2^{i} r_{n}^{\prime}} \leq \frac{r_{n}^{\prime}}{4},
\end{gathered}
$$

thus in view of $(10)$

$$
\rho\left(f_{n}, g_{n}\right) \leq \rho\left(f_{n}, g_{n 0}\right)+\rho\left(g_{n 0}, g_{n}\right) \leq \frac{r_{n}^{\prime}}{2}
$$

We have $0<a_{M}<1<3 j$, thus $\frac{r_{n}^{\prime}}{2}>\frac{a_{M} r_{n}^{\prime}}{6 j}$. Then putting $\delta_{n}=\frac{a_{M} r_{n}^{\prime}}{6 j}$ we get by (12) that $r_{n}^{\prime}-\rho\left(f_{n}, g_{n}\right) \geq \frac{r_{n}^{\prime}}{2}>\delta_{n}$, so

$$
B_{\rho}\left(g_{n}, \delta_{n}\right) \subset B_{\rho}\left(f_{n}, r_{n}^{\prime}\right) .
$$

Choose $h \in \tilde{A}_{i, j}(M)$ arbitrarily. According to (11) we have

$$
\begin{gathered}
\rho\left(h, g_{n}\right) \geq \frac{1}{2^{i} \mu\left(X_{i}\right)} \int_{h^{-1}(M) \cap X_{i}}^{*} \frac{\left|h-g_{n}\right|}{1+\left|h-g_{n}\right|} d \mu^{*} \geq \\
\geq \frac{1}{2^{i} \mu\left(X_{i}\right)} \mu^{*}\left(h^{-1}(M) \cap X_{i}\right) \cdot \frac{\min _{1 \leq k \leq m_{n}} r_{n k}}{1+\min _{1 \leq k \leq m_{n}} r_{n k}} \geq \frac{1}{2^{i} \mu\left(X_{i}\right)} \cdot \frac{\mu\left(X_{i}\right)}{j} \cdot \frac{a_{M} \eta_{n}}{1+a_{M} \eta_{n}}> \\
>\frac{1}{2^{i} j} \cdot \frac{a_{M} \eta_{n}}{1+\eta_{n}}=\delta_{n} .
\end{gathered}
$$

It means by (13) that $B_{\rho}\left(g_{n}, \delta_{n}\right) \subset B_{\rho}\left(f_{n}, r_{n}^{\prime}\right) \backslash \tilde{A}_{i, j}(M)$. Then in virtue of (9) we get $B_{\rho}\left(g_{n}, \delta_{n}\right) \subset B_{\rho}\left(f, r_{n}\right) \backslash\left(F \cup \tilde{A}_{i, j}(M)\right)$. Consequently

$$
\gamma\left(f, r_{n}, F \cup \tilde{A}_{i, j}(M)\right) \geq \delta_{n}>\frac{a_{M} \alpha r_{n}}{6 j}
$$

which justifies the porosity of $F \cup \tilde{A}_{i, j}(M)$ at $f$.

Theorem 2. Let $M$ be a $\sigma$-very porous set. Then $A\left(\chi_{\mathbb{R} \backslash M}\right)$ is $\sigma$-superporous in $(\mathcal{M}, \rho)$.

Proof. We may already suppose that $M=\bigcup_{k=1}^{\infty} M_{k}$, where $M_{k}$ is globally very porous and $a_{M_{k}}<1$ for all $k \in \mathbb{N}$.

Choose $f \in A\left(\chi_{\mathbb{R} \backslash M}\right)$. Then we have 


$$
\begin{gathered}
+\infty=\mu(X)-\int_{X}^{*}\left|\chi_{\mathbb{R} \backslash M} \circ f\right| d \mu^{*}=\mu(X)-\mu^{*}\left(f^{-1}(\mathbb{R} \backslash M)\right) \leq \\
\leq \mu^{*}\left(f^{-1}(M)\right) \leq \sum_{i, k \in \mathbb{N}} \mu^{*}\left(f^{-1}\left(M_{k}\right) \cap X_{i}\right),
\end{gathered}
$$

thus $\mu^{*}\left(f^{-1}\left(M_{k}\right) \cap X_{i}\right)>0$ for some $i, k \in \mathbb{N}$. It suffices now to pick $j \in \mathbb{N}$ such that $\mu^{*}\left(f^{-1}\left(M_{k}\right) \cap X_{i}\right) \geq \frac{\mu\left(X_{i}\right)}{j}$ then clearly $f \in \tilde{A}_{i, j}\left(M_{k}\right)$, consequently

$$
A\left(\chi_{\mathbb{R} \backslash M}\right) \subset \bigcup_{i, j, k \in \mathbb{N}} \tilde{A}_{i, j}\left(M_{k}\right),
$$

which concludes the proof by Lemma 4 .

Now we turn to characterizing the meagerness of $A\left(\chi_{\mathbb{R} \backslash M}\right)$ in $(\mathcal{M}, \rho)$ in terms of properties of $M$. We will need the following

Lemma 5. If $M$ is meager at some point of $\mathbb{R}$, then $A_{i, j}(M)$ is meager at some point of $\left(\mathcal{M}_{i}, \rho_{i}\right)$ for all $i, j \in \mathbb{N}$.

Proof. In the sequel we will use that the topology induced by $\rho_{i}$ on $\mathcal{M}_{i}$ is equivalent with the topology of convergence in measure on $X_{i}$, i.e. with the topology induced by the metric $m_{i}=\left.m\right|_{\mathcal{M}_{i} \times \mathcal{M}_{i}}$ (see [Ha], Problem $42(4)$ ).

Suppose that there exists an interval $U=B\left(t_{0}, r\right)\left(t_{0} \in \mathbb{R}, r>0\right)$ such that $U \cap M=\bigcup_{k=1}^{\infty} M_{k}$ for some nowhere dense sets $M_{k} \subset \mathbb{R}(k \in \mathbb{N})$. Without loss of generality we may assume that $M_{k} \subset M_{k+1}$ for all $k \in \mathbb{N}$. Let $f_{0} \equiv t_{0}$ on $X_{i}$ and put $V=B_{m_{i}}\left(f_{0}, r\right)$.

We will show that $V \cap A_{i, j}\left(M_{k}\right)$ is nowhere dense in $\left(\mathcal{M}_{i}, m_{i}\right)$ : take an open ball $B_{m_{i}}(f, \varepsilon)$ in $\mathcal{M}_{i}$. We may already suppose that $f \in V$ and $f$ equals a simple function $\sum_{s=1}^{m} b_{s} \chi_{D_{s}}$ where $b_{1}, \ldots, b_{m} \in U$ and $D_{1}, \ldots, D_{m}$ is a measurable partition of $X_{i}$.

Then the nowhere density of $M_{k}$ in $\mathbb{R}$ yields some $b_{s}^{\prime} \in \mathbb{R}$ and $0<\varepsilon_{0}<\frac{\mu\left(X_{i}\right)}{j}$ such that

$$
B\left(b_{s}^{\prime}, \varepsilon_{0}\right) \subset B\left(b_{s}, \varepsilon\right) \backslash M_{k} \text { for any } 1 \leq s \leq m .
$$

Choose $g \in B_{m_{i}}\left(f_{1}, \varepsilon_{0}\right)$ where $f_{1}=\sum_{s=1}^{m} b_{s}^{\prime} \chi_{D_{s}}$ then by (14)

$$
g^{-1}\left(M_{k}\right) \subset\left\{x \in X_{i} ;\left|f_{1}(x)-g(x)\right| \geq \varepsilon_{0}\right\} .
$$

Therefore $\mu^{*}\left(g^{-1}\left(M_{k}\right)\right) \leq \varepsilon_{0}<\frac{\mu\left(X_{i}\right)}{j}$, so $g \notin A_{i, j}\left(M_{k}\right)$. On the other hand $f_{1} \in$ $B_{m_{i}}(f, \varepsilon)$; thus,

$$
\emptyset \neq B_{m_{i}}(f, \varepsilon) \cap B_{m_{i}}\left(f_{1}, \varepsilon_{0}\right) \subset B_{m_{i}}(f, \varepsilon) \backslash A_{i, j}\left(M_{k}\right),
$$

which justifies the nowhere density of $V \cap A_{i, j}\left(M_{k}\right)$ in $\mathcal{M}_{i}$.

Finally, denote $V_{0}=B_{m_{i}}\left(f_{0}, r_{0}\right)$ where $r_{0}=\min \left\{r, \frac{1}{j}\right\}$. Pick $h \in A_{i, j}(M) \cap V_{0}$. Then $h^{-1}(M \backslash U) \subset\left\{x \in X_{i} ;\left|h(x)-f_{0}(x)\right| \geq r_{0}\right\}$, so $\mu^{*}\left(h^{-1}(M \backslash U)\right) \leq r_{0} \leq \frac{1}{j}<$ $\frac{\mu\left(X_{i}\right)}{2 j}$. Furthermore in view of the regularity of $\mu^{*}$ we get (cf. [F], Section 2.1.5(1))

$$
\begin{gathered}
\frac{\mu\left(X_{i}\right)}{j} \leq \mu^{*}\left(h^{-1}(M)\right) \leq \mu^{*}\left(h^{-1}(M \cap U)\right)+\mu^{*}\left(h^{-1}(M \backslash U)<\right. \\
<\lim _{k \rightarrow \infty} \mu^{*}\left(h^{-1}\left(M_{k}\right)\right)+\frac{\mu\left(X_{i}\right)}{2 j},
\end{gathered}
$$


hence $\lim _{k \rightarrow \infty} \mu^{*}\left(h^{-1}\left(M_{k}\right)\right)>\frac{\mu\left(X_{i}\right)}{2 j}$, so $h \in A_{i, 2 j}\left(M_{k}\right) \cap V_{0} \subset A_{i, 2 j}\left(M_{k}\right) \cap V$ for some $k \in \mathbb{N}$. Therefore

$$
A_{i, j}(M) \cap V_{0} \subset \bigcup_{k=1}^{\infty} A_{i, 2 j}\left(M_{k}\right) \cap V
$$

which means that $A_{i, j}(M)$ is meager at $f_{0}$ in $\mathcal{M}_{i}$.

Theorem 3. If $M$ is meager at some point of $\mathbb{R}$ then $A\left(\chi_{\mathbb{R} \backslash M}\right)$ is meager in $(\mathcal{M}, \rho)$.

Proof. Let $t_{0} \in \mathbb{R}$ and $r>0$ be such that $B\left(t_{0}, r\right) \cap M$ is meager in $\mathbb{R}$. Let $V_{i}=B_{m_{i}}\left(f_{0}, r_{0}\right)$ where $f_{0} \equiv t_{0}$ on $X$ and $0<r_{0}=\min \left\{r, \frac{1}{2}\right\}$. Then by Lemma 5 $A_{i, 2}(M) \cap V_{i}$ is meager in $\left(\mathcal{M}_{i}, \rho_{i}\right)$ for all $i \in \mathbb{N}$.

Choose $f \in A\left(\chi_{\mathbb{R} \backslash M}\right)$. Then $\mu^{*}\left(f^{-1}(\mathbb{R} \backslash M)\right)<+\infty$ and by the regularity of $\mu^{*}$ there exists a $\mu^{*}$-hull $B$ of $f^{-1}(\mathbb{R} \backslash M)$ (see $[\mathrm{F}]$, Section 2.1.4). Consequently, $\mu\left(B \cap X_{i}\right)=\mu^{*}\left(f^{-1}(\mathbb{R} \backslash M) \cap X_{i}\right)=\mu^{*}\left(X_{i} \backslash\left(X_{i} \cap f^{-1}(M)\right)\right)$; thus,

$$
+\infty>\mu^{*}\left(f^{-1}(\mathbb{R} \backslash M)\right)=\mu(B)=\sum_{i=1}^{\infty} \mu\left(B \cap X_{i}\right)=\sum_{i=1}^{\infty} \mu^{*}\left(X_{i} \backslash\left(X_{i} \cap f^{-1}(M)\right)\right) .
$$

Then for all $i \geq m(m \in \mathbb{N})$ we have

$$
\frac{\mu\left(X_{i}\right)}{2}>1>\mu^{*}\left(X_{i} \backslash\left(X_{i} \cap f^{-1}(M)\right)\right) \geq \mu\left(X_{i}\right)-\mu^{*}\left(X_{i} \cap f^{-1}(M)\right),
$$

hence $\left.f\right|_{X_{i}} \in A_{i, 2}(M)$ for all $i \geq m$. Accordingly,

$$
A\left(\chi_{\mathbb{R} \backslash M}\right) \subset \bigcup_{m=1}^{\infty} P_{m} \text { where } P_{m}=\Pi_{i=1}^{m-1} \mathcal{M}_{i} \times \Pi_{i=m}^{\infty} A_{i, 2}(M) \text { for each } m \in \mathbb{N} .
$$

It suffices now to show by Remark 1 that $P_{m}$ is meager in $P=\Pi_{i=1}^{\infty} \mathcal{M}_{i}$ for every $m \in \mathbb{N}$ : Let $\mathbf{U}=\Pi_{i=1}^{n} U_{i} \times \Pi_{n+1}^{\infty} \mathcal{M}_{i}$ be any basic open set of the product topology on $P$ such that $n \geq m$. Denote by $\mathbf{V}$ the open set $\Pi_{i=1}^{n} U_{i} \times V_{n+1} \times \Pi_{n+2}^{\infty} \mathcal{M}_{i} \subset P$. Then $\mathbf{V} \subset \mathbf{U}$ and $\mathbf{V} \cap P_{m} \subset \Pi_{i=1}^{n} U_{i} \times\left(V_{n+1} \cap A_{n+1,2}(M)\right) \times \Pi_{i=n+2}^{\infty} A_{i, 2}(M)$, which is meager in $P$. It means by Theorem 1.7. in [HMC] that $P_{m}$ is meager in $P$.

Corollary. $A\left(\chi_{\mathbb{R} \backslash M}\right)$ is meager in $\left(s, \rho_{F}\right)$ if and only if $M$ is meager at some point of $\mathbb{R}$.

Proof. The sufficiency follows from the previous theorem by putting $X=\mathbb{N}, S=$ $\mathcal{P}(\mathbb{N})$ and the counting measure on $\mathbb{N}$ for $\mu$.

Conversely, suppose that $M$ is non-meager everywhere in $\mathbb{R}$. Then $M$ with the relative topology is a dense Baire subspace of $\mathbb{R}$. Then the product $E=M^{\mathbb{N}}$ is a Baire space which is clearly dense in $s$ ([HMC], Lemma 5.6.). Therefore $E$ is non-meager in $s$ and hence $A\left(\chi_{\mathbb{R} \backslash M}\right) \supset E$ is non-meager in $s$.

Remark 2. In connection with the Corollary a question arises if a similar characterization of $A\left(\chi_{\mathbb{R} \backslash M}\right)$ is possible also in $\mathcal{M}$. Mimicking the above proof and using Remark 1 it would be sufficient to prove that non-meagerness of $M$ everywhere in $\mathbb{R}$ implies non-meagerness of $A_{i, 0}$ everywhere in $\mathcal{M}_{i}$ for each $i \in \mathbb{N}$, further that $\mathcal{M}_{i}$ is separable for each $i \in \mathbb{N}$. This last condition is needed for the theorem on product of Baire spaces ([HMC], Lemma 5.6.), thus we may consider the question only for separable measure spaces $(X, S, \mu)$ (see [Ha], §41). 
It is not hard to show that this is really the case if each $X_{i}$ is a finite disjoint sum of atoms, however in general the answer is not known to me.

Remark 3. Another question here arises in connection with finding necessary conditions for $\sigma$-porosity of $A\left(\chi_{\mathbb{R} \backslash M}\right)$ in $\mathcal{M}$ (or at least in $s$ ). If we want to use some argument similar to that of in the Corollary, we would need some "porosity-Baire" product theorem as the mentioned result of Oxtoby ([O1],[HMC]). This ultimately breaks down to proving a porosity version of the well-known Kuratowski-Ulam theorem on sections of nowhere dense subsets of the product space $([\mathrm{O} 2]$, Theorem 15.1). More precisely, the questions are as follows:

(i) If $X$ and $Y$ are separable metric spaces and $E$ is a porous subset of $X \times Y$ with (say) the box metric, then are the $x$-sections $E_{x}$ of $E$ porous in $Y$ except for a $\sigma$-porous set in $X$ ?

(ii) Call a metric space $Z$ p-Baire if every nonempty open subset of $Z$ is non- $\sigma$ porous. Is the property of being separably p-Baire (countably) productive?

The preceding theorems provide sufficient background for investigating the class

$$
\mathcal{U}=\{\Phi \in \mathcal{F} ; A(\Phi) \text { is } \sigma \text {-superporous in }(\mathcal{M}, \rho)\} .
$$

Theorem 4. We have

(i) $\operatorname{card}\left(\mathcal{U} \cap \mathcal{F}_{m}\right)=\operatorname{card} \mathcal{U}=2^{c}$

(ii) $\operatorname{card}(\mathcal{F} \backslash \mathcal{U})=2^{c}$ for $\left(s, \rho_{F}\right)$.

Proof. (i) Every subset of the Cantor's ternary set $C$ is very porous therefore in view of Theorem $2 \chi_{\mathbb{R} \backslash E} \in \mathcal{U} \cap \mathcal{F}_{m}$ for every $E \subset C$, further $\chi_{\mathbb{R} \backslash E} \neq \chi_{\mathbb{R} \backslash E^{\prime}}$ provided $E \neq E^{\prime}$. Consequently $\operatorname{card}\left(U \cap \mathcal{F}_{m}\right) \geq \operatorname{card} \mathcal{P}(C)=2^{c}$. Further clearly $\operatorname{card} \mathcal{U} \leq \operatorname{card} \mathcal{F} \leq \operatorname{card}\left(\mathbb{R}^{\mathbb{R}}\right)=2^{c}$.

(ii) If we restrict ourselves to $\left(s, \rho_{F}\right)$ only, then $\chi_{E} \notin \mathcal{U}$ for each $E \subset C$ since $A\left(\chi_{E}\right)=s \backslash A\left(\chi_{\mathbb{R} \backslash E}\right)$ and $\left(s, \rho_{F}\right)$ is a nonmeager space by Lemma 2. Thus again $2^{c}=\operatorname{card} \mathcal{P}(C) \leq \operatorname{card}(\mathcal{F} \backslash \mathcal{U}) \leq \operatorname{card} \mathcal{F} \leq 2^{c}$.

Further we have

Theorem 5. $\mathcal{U}$ is residual in $\mathcal{F}$.

Proof. See [TZs], Lemma 2 and our Theorem 1.

Remark 4. It is worth noticing that if we restrict our investigations onto $\mathcal{F}_{m}$ only, then similar results hold. Actually, Lemma 3-4 and Theorem 1-2 hold without change, we need only to replace $\mu^{*}$ by $\mu$ and the upper integral by integral, respectively in the proofs.

We can also prove the analogue of Tóth's Theorem (Theorem 5) for $\mathcal{F}_{m}$ :

Theorem 5'. $\mathcal{U} \cap \mathcal{F}_{m}$ is residual in $\left(\mathcal{F}_{m}, d\right)$.

Proof. See Lemma 2 in [TZs]. The only difference is in proving the density of $\mathcal{U}_{0}=\left\{\Phi \in \mathcal{F}_{m} ; \Phi\right.$ satisfies (6) for some $\left.t_{0} \in \overline{\mathbb{R}}\right\}$ in $\left(\mathcal{F}_{m}, d\right)$, more precisely in proving that $\Psi=\Phi \chi_{M}+\frac{\varepsilon}{4} \chi_{\mathbb{R} \backslash M} \in \mathcal{F}_{m}$, where $\Phi \in \mathcal{F}_{m}, \varepsilon>0$ and $M=\{t \in$ $\mathbb{R}$; either $t \notin(0,1)$ or $t \in(0,1)$ and $\left.|\Phi(t)| \geq \frac{\varepsilon}{4}\right\}$. 
To show this pick $f \in \mathcal{M}, c \in \mathbb{R}$ arbitrarily and observe that

$$
\begin{aligned}
& (\Psi \circ f)^{-1}([c,+\infty))=\left\{\begin{array}{l}
(\Phi \circ f)^{-1}([c,+\infty)), \text { if } c>\frac{\varepsilon}{4} \\
(\Phi \circ f)^{-1}([c,+\infty)) \cup\left(f^{-1}((0,1)) \cap(\Phi \circ f)^{-1}\left(\left(-\frac{\varepsilon}{4}, \frac{\varepsilon}{4}\right)\right)\right),
\end{array}\right. \\
& \text { if } c \leq \frac{\varepsilon}{4}
\end{aligned}
$$

thus $\Psi \circ f \in \mathcal{M}$.

\section{REFERENCES}

[AB] S. Agronsky - A. Bruckner, Local compactness and porosity in metric spaces, Real Analysis Exchange 11 (1985-86), 365-379.

[D] E.P. Dolženko, Boundary properties of arbitrary functions, Izv. Akad. Nauk SSSR Ser. Mat. 31 (1967), 3-14 (Russian).

[F] H. Federer, Geometric Measure Theory, Springer-Verlag, Berlin-Heidelberg-New York, 1969.

[G] J.I. Gribanov, On metrization of a space of functions, CMUC 4 (1963), 43-46 (Russian).

[Ha] P.R. Halmos, Measure Theory, D. van Nostrand, Toronto-New York-London, 1950.

[HMC] R.C. Haworth - R.A. McCoy, Baire spaces, Dissertationes Math. 141 (1977).

[K] J.L. Kelley, General Topology, Springer-Verlag, New York, 1984.

[KG] A.A. Kirillov - A.D. Gvishiani, Theorems and Problems in Functional Analysis, Springer, New York, 1982.

[O1] J.C. Oxtoby, Cartesian products of Baire spaces, Fund. Math. 49 (1961), 157-166.

[O2] J.C. Oxtoby, Measure and Category, Springer, New York, 1971.

[Re] D.L. Renfro, A Study of Porous and $\sigma$-Porous Sets, preprint.

[TZs] J. Tóth - L. Zsilinszky, On a typical property of functions, Math. Slovaca 45 (1995), $121-127$.

[W] W. Wilczyǹski, A category analogue of the density topology, approximate continuity and the approximate derivative, Real Analysis Exchange 10 (1984-85), 241-265.

[Za1] L. Zajíček, Porosity and $\sigma$-porosity, Real Analysis Exchange 13 (1987-88), 314-350.

[Za2] L. Zajíček, Porosity, I-density topology and abstract density topologies, Real Analysis Exchange 12 (1986-87), 313-326.

[Za3] L. Zajíček, Sigma-porous sets in topologically complete metric spaces, preprint.

[Zs1] L. Zsilinszky, On subspaces of measurable real functions, Portugaliae Math. 52 (1995), $125-130$

[Zs2] L. Zsilinszky, On normability of a space of measurable real functions, Acta Math. et Inf. 2 (1995), 73-76. 